\title{
Necessidades Físicas, Emocionais e Socioeconômicas no Pós-tratamento do Câncer de Cabeça e Pescoço: um Estudo Qualitativo
}

doi: https://doi.org/10.32635/2176-9745.RBC.2021v67n3.1221

\author{
Physical, Emotional and Socioeconomic Needs in the Post-Treatment of Head and Neck Cancer: a Qualitative Study \\ Necesidades Físicas, Emocionales y Socioeconómicas en el Postratamiento del Cáncer de Cabeza y Cuello: un Estudio \\ Cualitativo
}

Carolina de Menezes Rabello'; Rildo Pereira da Silva²; Antonio Tadeu Cheriff dos Santos³; Fernando Lopes Tavares de Lima; Liz Maria de Almeida ${ }^{5}$

RESUMO

Introduçáo: Os pacientes com câncer de cabeça e pescoço constituem um grupo com necessidades complexas pouco estudadas na fase de pós-tratamento. Objetivo: Compreender a experiência e as necessidades da fase de sobrevivência ao câncer de cabeça e pescoço a partir da perspectiva dos próprios pacientes, seus familiares, e profissionais de saúde de serviços de oncologia. Método: Pesquisa qualitativa, exploratória descritiva, tendo como referencial metodológico a hermenêutica-dialética. Adotaram-se, como técnicas de produção de dados, a entrevista semiestruturada e o grupo focal. Resultados: O material empírico foi organizado em três categorias de necessidades físicas, emocionais e socioeconômicas e estruturado em três abrangentes sentidos envolvendo as consequências do impacto biológico, psíquico e socioeconômico da doença e seu tratamento. Destacam-se, para o primeiro, os aspectos relativos às adaptaçóes do hábito alimentar, comunicaçấo e manejo dos efeitos do tratamento; o apoio emocional para pacientes e familiares no segundo; e as informaçóes sobre benefícios sociais para o terceiro. Conclusáo: As necessidades físicas, emocionais e socioeconômicas da doença e seu tratamento se entrelaçam para formar uma complexa rede de desafios para as políticas públicas de controle do câncer no Brasil.

Palavras-chave: Determinação de Necessidades de Cuidados de Saúde; Necessidades e Demandas de Serviços de Saúde; Neoplasias de Cabeça e Pescoço; Oncologia; Sobrevida.

\section{ABSTRACT}

Introduction: Head and neck cancer patients are a group with complex needs barely studied in the post-treatment phase. Objective: To understand the experience and needs of the head and neck cancer survival phase from the perspective of the patients themselves, their families, and health professionals from oncology services. Method: Qualitative, exploratory, and descriptive study, using the hermeneutic-dialectic methodological framework. Semi-structured interviews and focus group were adopted as data production techniques. Results: The empirical material was organized into three categories of needs physical, emotional and socioeconomic and structured in three broad senses involving the consequences of the biological, psychological, and socioeconomic impact of the disease and its treatment. The aspects related to the adaptations of eating habits, communication and management of the treatment effects stand out for the first; emotional support for patients and families in the second, and information about social benefits for the third impact. Conclusion: The physical, emotional, and socioeconomic needs of the disease and its treatment are intertwined to form a complex network of challenges for public cancer control policies in Brazil. Key words: Needs Assessment; Health Services Needs and Demand; Head and Neck Neoplasms; Medical Oncology; Survival.

\section{RESUMEN}

Introducción: Los pacientes con cáncer de cabeza y cuello son un grupo con necesidades complejas poco estudiadas en la fase postratamiento. Objetivo: Conocer la experiencia y necesidades de la fase de supervivencia del cáncer de cabeza y cuello desde la perspectiva de los propios pacientes, sus familias y los profesionales sanitarios de los servicios de oncología. Método: Investigación cualitativa, exploratoria y descriptiva, utilizando el marco metodológico hermenéutico-dialéctico. Se adoptaron las entrevistas semiestructuradas y el grupo focal como técnicas de producción de datos. Resultados: El material empírico se organizó en tres categorías de necesidades físicas, emocionales y socioeconómicas y estructurado en tres amplios sentidos que involucran las consecuencias del impacto biológico, psicológico y socioeconómico de la enfermedad y su tratamiento. Los aspectos relacionados con la adaptación de los hábitos alimentarios, la comunicación y el manejo de los efectos del tratamiento destacan por el primero; apoyo emocional a pacientes y familiares en el segundo; e información sobre beneficios sociales para el tercero. Conclusión: Las necesidades físicas, emocionales y socioeconómicas de la enfermedad y su tratamiento se entrelazan para formar una compleja red de desafíos para las políticas públicas de control del cáncer en Brasil. Palabras clave: Evaluación de Necesidades; Necesidades y Demandas de Servicios de Salud; Neoplasias de Cabeza y Cuello; Oncología Médica; Sobrevida.

1-5Instituto Nacional de Câncer José Alencar Gomes da Silva (INCA). Rio de Janeiro (RJ), Brasil.

1Programa de Residência Multiprofissional em Oncologia. E-mail: rabellocm@gmail.com. Orcid iD: https://orcid.org/0000-0001-5417-4055

${ }^{2}$ Coordenação de Pesquisa/Comitê de Ética em Pesquisa. E-mail: rildo@inca.gov.br. Orcid iD: https://orcid.org/0000-0001-6818-4748

${ }^{3}$ Divisão de Pesquisa Populacional/Núcleo de Pesquisa e Estudos Qualitativos. E-mail: cheriff@inca.gov.br. Orcid iD: https://orcid.org/0000-0002-3577-0772

${ }^{4}$ Coordenação de Ensino. E-mail: flima@inca.gov.br. Orcid iD: https://orcid.org/0000-0002-8618-7608

${ }^{5}$ Coordenação de Prevenção e Controle do Câncer. E-mail: Ialmeida@inca.gov.br. Orcid iD: https://orcid.org/0000-0002-2359-0651

Endereço para correspondência: Antonio Tadeu Cheriff dos Santos. Rua Marques de Pombal, 125, $7{ }^{\circ}$ andar - Centro. Rio de Janeiro (RJ), Brasil. CEP $20.230-240$. 


\section{INTRODUÇÃO}

O câncer de cabeça e pescoço ocupa o quinto lugar na incidência global de câncer ${ }^{1}$. No Brasil, para cada ano do triênio 2020-2022, considerando os tumores de lábios, cavidade oral, faringe, laringe, cavidade nasal e tireoide, as estimativas apontam a ocorrência de 36.620 casos novos, representando a terceira maior incidência entre homens e mulheres ${ }^{2}$.

No mundo, em decorrência do aumento da incidência em pacientes mais jovens, bem como meios de diagnóstico aprimorados e escolhas terapêuticas mais eficazes, as taxas de sobrevivência vêm significativamente melhorando. Entretanto, em longo prazo, essa sobrevivência é acompanhada de morbidades e sequelas, em razão, principalmente, dos tipos de tratamentos dispensados ${ }^{3}$. No Brasil, a situação é similar, embora agravada pelo fato de os diagnósticos serem realizados de forma tardia, o que aumenta a possibilidade de morbidade e sequelas físicas, psicológicas e sociais ${ }^{4}$.

Os pacientes com câncer de cabeça e pescoço constituem um grupo com necessidades complexas no pós-tratamento. A experiência da sobrevivência desses pacientes e de outros deve ser entendida como uma jornada, não só de busca da cura e/ou tratamento, mas também de compreensão e mitigação dos impactos clínicos, emocionais e sociais de todo um processo de tratamento, desde o seu diagnóstico até os cuidados de fim de vida ${ }^{5}$.

Como pacientes crônicos, esses indivíduos demandam um projeto terapêutico complexo e em longo prazo, no qual devem participar profissionais de saúde de múltiplas formaçôes. Para pacientes que se encontram na fase de pós-tratamento, a cronicidade não está relacionada apenas com o histórico do câncer, mas também com a toxicidade dos tratamentos dispensados ${ }^{6}$.

As principais comorbidades físicas, emocionais e sociais que atingem esse grupo são: depressão e retração social por alteração da autoimagem; dificuldades de comer e beber (incluindo disfagia, problemas dentários, trismo e perturbação sensorial); hipotireoidismo; maior possibilidade de incidência de segunda malignidade e recidivas; dependência de álcool e nicotina; e perda de rendimentos ${ }^{6-8}$. As estratégias destinadas a minimizar essas complicaçóes e otimizar seu gerenciamento em longo prazo são cada vez mais necessárias ${ }^{3}$.

Muito embora existam recomendaçôes e propostas de desenvolvimento de pesquisa e planos de cuidados para identificação de necessidades e coordenação do atendimento dos sobreviventes, nas esferas assistenciais especializadas e primárias ${ }^{9-12}$, a efetividade e alcance dessas medidas ainda necessitam de uma análise mais aprimorada ${ }^{13-16}$. Faltam, também, estudos relacionados aos fatores socioeconômicos ${ }^{6} \mathrm{e}$ informaçóes e pesquisas sobre as necessidades e a qualidade dos cuidados e suporte de longo prazo ${ }^{15,16}$. No Brasil, apesar da existência dos estudos voltados para protocolos clínicos e terapêuticos, bem como para a qualidade de vida ${ }^{4,17}$, não há uma discussão sistemática e ampliada acerca de planos de cuidados, políticas públicas e linhas de pesquisa voltadas para as necessidades, em longo prazo, de sobreviventes do câncer ao cabeça e pescoço.

Tendo em vista esse cenário, o objetivo deste estudo é compreender a experiência e as necessidades da fase de sobrevivência ao câncer de cabeça e pescoço a partir das perspectivas dos próprios pacientes, seus familiares e profissionais de saúde de serviços em oncologia.

\section{MÉTODO}

Trata-se de pesquisa de natureza qualitativa exploratória, norteada pelo referencial teórico-metodológico da hermenêutica-dialética em sua dupla função: configuração das categorias e das questóes relacionadas ao objeto e como procedimento de análise do material empírico ${ }^{18-20}$.

A união da hermenêutica com a dialética foi fecunda na condução de um processo ao mesmo tempo compreensivo e crítico das atribuições simbólicas à experiência da sobrevivência ao câncer tanto na perspectiva dos pacientes e familiares que experenciam o adoecimento e as formas de tratamento e de prestação de serviços médicos quanto dos profissionais de saúde que, por sua vez, protagonizam a aplicação de programas clínico-assistenciais e gerenciam políticas públicas de saúde.

Empregou-se amostragem por conveniência e intencional de seis sobreviventes de câncer de cabeça e pescoço, com diagnóstico confirmado há pelo menos 12 meses, três familiares de sobreviventes de câncer de cabeça e pescoço, cinco enfermeiros com, pelo menos, um ano de experiência profissional e dois médico-gerentes de serviços de oncologia clínica ou cirúrgica para tratamento do câncer de cabeça e pescoço.

A seleção dos pacientes e familiares baseou-se nas taxas dos quatro tipos de tumores de cabeça e pescoço mais incidentes no Brasil ${ }^{2}$, sendo um de tireoide, um de laringe e os demais de cavidade oral. Os pacientes que participaram do estudo possuíam condiçóes físico-clínicas que lhes permitiam expressão verbal. Os sobreviventes e familiares não guardavam relaçôes de parentesco.

Este estudo foi aprovado por um Comitê de Ética em Pesquisa sob o número de protocolo 660.486. Todos os participantes foram orientados a respeito dos objetivos e métodos e forneceram o consentimento por meio do Termo de Consentimento Livre e Esclarecido (TCLE). A 
todos os participantes do estudo, foram atribuídos códigos para a proteção da sua identidade: (S) para sobreviventes; (F) para familiares; (E) para enfermeiros; e (G) para gestores-médicos.

Foram realizadas entrevistas semiestruturadas com os sobreviventes, familiares e gestores. No caso dos enfermeiros, realizou-se um grupo focal objetivando compreender os consensos e dissensos a respeito do objeto de estudo.

As entrevistas e o grupo focal foram conduzidos por três pesquisadores com experiência prévia, entre junho e outubro de 2014, em um centro de oncologia no Rio de Janeiro, Brasil. A construção e a coleta dos dados foram realizadas nessa ordem: sobreviventes, familiares, gestores e enfermeiros.

Tanto para as entrevistas quanto para o grupo focal, foi utilizado um roteiro de tópicos sobre as experiências, dificuldades e necessidades da fase de sobrevivência ao câncer de cabeça e pescoço. O tempo médio de cada entrevista foi de 50 minutos com sobreviventes e membros da família e de 30 minutos com os médico-gestores. O grupo focal durou 90 minutos. Todo o processo de construção e coleta dos dados foi gravado e transcrito para análise.

A análise hermenêutico-dialética seguiu a orientação de Minayo ${ }^{18}$ e Gomes $^{19}$. A hermenêutica é a busca de compreensão de sentido que se dá na comunicação entre seres humanos, tendo na linguagem seu núcleo central. A dialética busca, nos fatos, na linguagem, nos símbolos e na cultura, os núcleos obscuros e contraditórios para realizar uma crítica sobre eles.

O conteúdo da narrativa de cada participante foi analisado considerando que a experiência de sobrevivência do câncer implica na interpenetração das dimensóes biológica, emocional, econômica e social ${ }^{21}$ e exige uma abordagem interpretativa e crítica para o fenômeno ${ }^{19,22,23}$. Esse processo envolveu uma coconstruçáo de significados, uma vez que as falas dos participantes e a interpretação dos pesquisadores estavam envolvidas em um círculo hermenêutico de compreensão e interpretação críticas à linguagem e à escrita ${ }^{20,24-26}$.

A análise seguiu as cinco etapas preconizadas ${ }^{18,22}$ : (a) leitura compreensiva; (b) identificação dos sentidos subjacentes às falas dos participantes; (c) problematização das ideias e dos sentidos presentes nos depoimentos e articulação com significados socioculturais; (d) elaboração de síntese entre os dados empíricos e as informaçóes provenientes de estudos acerca da temática da sociologia do câncer, sobrevivência ao câncer, em especial ao câncer de cabeça e pescoço, oferecendo aportes teóricos; (e) elaboração de esquemas do processo de análise interpretativa, contendo categorias empíricas e suas correlaçốes com categorias teóricas, gerando sínteses de sentidos.

A interpretação hermenêutico-dialética do conteúdo do estudo foi compartilhada pelos pesquisadores que, em reunióes semanais, realizaram uma leitura detalhada e aprofundada das transcriçóes e identificaram os significados subjacentes ao material empírico, bem como configuraram e cotejaram as categorias empíricas e teóricas que constituíram os esquemas hermenêuticos e críticos utilizados para a análise interpretativa.

As categorias e as subcategorias empíricas foram elaboradas como um mosaico que, embora partindo e identificando a perspectiva dos participantes, se uniu em uma visão integrada e compreensiva acerca das necessidades dos sobreviventes ao câncer de cabeça e pescoço.

Os referenciais teóricos de análise eleitos foram: (1) a hermenêutica da saúde de Gadamer ${ }^{24}$ como reflexão sobre os elementos subjacentes à complexidade da saúde; (2) a noção de sobrevivência de Mullan ${ }^{5}$ como precursora da ruptura com a noção biomédica de sobrevivência; (3) orientaçôes e guias de tratamento acerca dos cuidados a serem ofertados aos sobreviventes de câncer de cabeça e pescoço ${ }^{7,9-11}$; e (4) a hermenêutica-dialética de Habermas ${ }^{27,28}$ como uma proposta metodológica de investigação social.

A análise baseada pelo princípio da saturação teórica e configurada a partir do conjunto das falas e da extração das suas respectivas interpretaçóes de significado ${ }^{18,19,23}$ resultou em três categorias empíricas.

\section{RESULTADOS}

O grupo dos sobreviventes participantes foi formado, em sua maioria, por homens, entre 64 e 79 anos, casados, aposentados, escolarizados com nível até o fundamental, possuindo familiares como dependentes financeiros. Tiveram como tratamento, majoritariamente, a associação de cirurgia e radioterapia (quatro participantes). Todos os pacientes ainda se encontravam em tratamento (entre 15 dias e dois anos), sendo que dois deles estavam em tratamento de uma recidiva.

Os três familiares, com de idades de 34, 50 e 53 anos, eram esposas de pacientes com diagnóstico câncer de cavidade oral.

O grupo de enfermeiras foi formado por profissionais especializados em oncologia, experiência profissional entre três e 30 anos e idade entre 28 e 56 anos. O grupo de médico-gestores foi composto por cirurgióes de cabeça e pescoço com um tempo de experiência profissional entre 30 e 35 anos e idade entre 58 e 60 anos.

Os resultados das entrevistas e do grupo focal foram organizados em três categorias empíricas: 
necessidades físicas; necessidades emocionais; e necessidades socioeconômicas. Cada categoria possui, respectivamente, subcategorias que se encontram listadas e definidas no Quadro 1. Os Quadros 2, 3 e 4 complementam os resultados do estudo apresentando, para cada categoria, o conjunto das subcategorias e os seus respectivos trechos de depoimentos selecionados.

A categoria necessidades físicas abrange o conjunto das necessidades originadas do impacto biológico da patologia e seu tratamento, com repercussóes no corpo físico e em suas respectivas exigências de manejo e acompanhamento clínico (Quadro 2).

As necessidades emocionais compreendem o conjunto originado pelo impacto psíquico da patologia e seu tratamento, com repercussóes no comportamento, nas emoçóes e em suas respectivas exigências de acompanhamento psicológico (Quadro 3).

As necessidades socioeconômicas são decorrentes do impacto socioeconômico da patologia e seu tratamento, com repercussóes nas finanças e na convivência social de pacientes e familiares (Quadro 4).

\section{DISCUSSÃO}

Os sentidos das falas nas três categorias, entre os diferentes grupos de depoentes, convergiram para a configuração de um mesmo cenário de necessidades.

\section{NECESSIDADES FÍSICAS}

A categoria necessidades físicas apresenta o sentido de necessidades como consequências do impacto biológico da doença e seu tratamento, remetendo ao comprometimento e ao desajuste de funções orgânicas e estruturas anatômicas, decorrentes da própria doença ou das intervençôes terapêuticas.

Os sobreviventes e familiares verbalizaram o forte e agudo impacto do adoecimento e do resultado do tratamento na vida de todos os envolvidos. São relatos

Quadro 1. Categorias e subcategorias empíricas - Definições

\begin{tabular}{|c|c|c|}
\hline $\begin{array}{l}\text { Categoria } \\
\text { empírica }\end{array}$ & Subcategorias & Definição das subcategorias empíricas \\
\hline \multirow[t]{3}{*}{$\begin{array}{l}\text { Necessidades } \\
\text { físicas }\end{array}$} & $\begin{array}{l}\text { Adaptações do hábito } \\
\text { alimentar }\end{array}$ & $\begin{array}{l}\text { Conjunto de adaptações dos costumes e modos de } \\
\text { alimentação, determinado pelo impacto biológico da patologia } \\
\text { e seu tratamento, com repercussões no processo digestivo }\end{array}$ \\
\hline & $\begin{array}{l}\text { Adaptações da } \\
\text { capacidade de } \\
\text { comunicação oral }\end{array}$ & $\begin{array}{l}\text { Conjunto de adaptações da capacidade de comunicação } \\
\text { oral, determinado pelo impacto biológico da patologia e seu } \\
\text { tratamento, com repercussões no processo fonatório }\end{array}$ \\
\hline & $\begin{array}{l}\text { Intervenções clínicas } \\
\text { relacionadas aos efeitos } \\
\text { físicos da doença e seu } \\
\text { tratamento }\end{array}$ & $\begin{array}{l}\text { Conjunto de intervenções clínicas referente ao manejo dos } \\
\text { efeitos decorrentes da patologia e do seu tratamento }\end{array}$ \\
\hline \multirow[t]{3}{*}{$\begin{array}{l}\text { Necessidades } \\
\text { emocionais }\end{array}$} & $\begin{array}{l}\text { Estratégias de } \\
\text { enfrentamento ante a } \\
\text { possibilidade de recidiva }\end{array}$ & $\begin{array}{l}\text { Conjunto de estratégias a ser efetivado por meio de } \\
\text { acompanhamento clínico e suporte psicológico, com vistas ao } \\
\text { enfrentamento da possibilidade de recidiva }\end{array}$ \\
\hline & $\begin{array}{l}\text { Suporte psicológico ante } \\
\text { o diagnóstico }\end{array}$ & $\begin{array}{l}\text { Conjunto de ações de suporte psicológico a ser efetivado } \\
\text { quando da comunicação do diagnóstico positivo }\end{array}$ \\
\hline & $\begin{array}{l}\text { Suporte psicológico } \\
\text { para a sobrevivência }\end{array}$ & $\begin{array}{l}\text { Conjunto de ações de suporte psicológico direcionado para a } \\
\text { superação dos traumas e obstáculos emocionais, com vistas a } \\
\text { motivação e a valorização da vida na etapa da sobrevivência }\end{array}$ \\
\hline \multirow[t]{2}{*}{$\begin{array}{l}\text { Necessidades } \\
\text { socioeconômicas }\end{array}$} & $\begin{array}{l}\text { Restabelecimento da } \\
\text { convivência social }\end{array}$ & $\begin{array}{l}\text { Conjunto das necessidades, originado do impacto social da } \\
\text { patologia e seu tratamento, com repercussões nas relações } \\
\text { sociais cotidianas e suas respectivas exigências de ações de } \\
\text { restabelecimento da convivência entre diferentes grupos } \\
\text { sociais }\end{array}$ \\
\hline & $\begin{array}{l}\text { Informações e } \\
\text { acessibilidade aos } \\
\text { direitos sociais }\end{array}$ & $\begin{array}{l}\text { Conjunto das necessidades, originado do impacto } \\
\text { socioeconômico da patologia e seu tratamento sobre o } \\
\text { sobrevivente, com repercussões na sua cidadania e suas } \\
\text { respectivas exigências de informação democratizada e acesso } \\
\text { real aos direitos e à equidade sociais }\end{array}$ \\
\hline
\end{tabular}


Quadro 2. Depoimentos das subcategorias da categoria empírica "necessidades físicas"

\begin{tabular}{|c|c|}
\hline $\begin{array}{c}\text { Subcategorias } \\
\text { empíricas }\end{array}$ & Trechos de depoimentos \\
\hline $\begin{array}{l}\text { Adaptações } \\
\text { do hábito } \\
\text { alimentar }\end{array}$ & 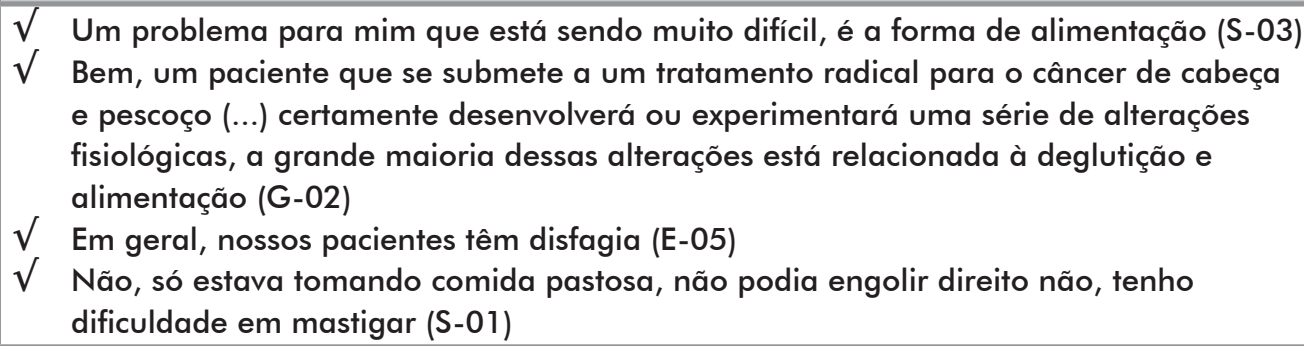 \\
\hline $\begin{array}{l}\text { Adaptações da } \\
\text { capacidade de } \\
\text { comunicação } \\
\text { oral }\end{array}$ & $\begin{array}{l}\sqrt{ } \begin{array}{l}\text { Raramente saio de casa. Se vou a um lugar muito barulhento, não consigo falar. Na } \\
\text { rua, mesmo usando o dispositivo, não consigo conversar por causa do barulho. Vou só }\end{array} \\
\text { para a casa das minhas filhas (...) Só saio se o local não precisar de conversa (S-04) } \\
\sqrt{ } \quad \begin{array}{l}\text { (...) a fono está sendo muito bem recebida pelo meu irmão, né? Porque ele ficou } \\
\text { mais depressivo, porque ele não podia se comunicar com as pessoas, ele tinha que }\end{array} \\
\sqrt{\sqrt{ }} \text { escrever (F-02) } \\
\sqrt{ } \text { Estou me sentindo um pouco, só rouca, não pode falar muito não (S-01) } \\
\text { isso aí sem sombra de dúvidas (E-04) }\end{array}$ \\
\hline $\begin{array}{l}\text { Intervenções } \\
\text { clínicas } \\
\text { relacionadas } \\
\text { aos efeitos } \\
\text { físicos da } \\
\text { doença e seu } \\
\text { tratamento }\end{array}$ & 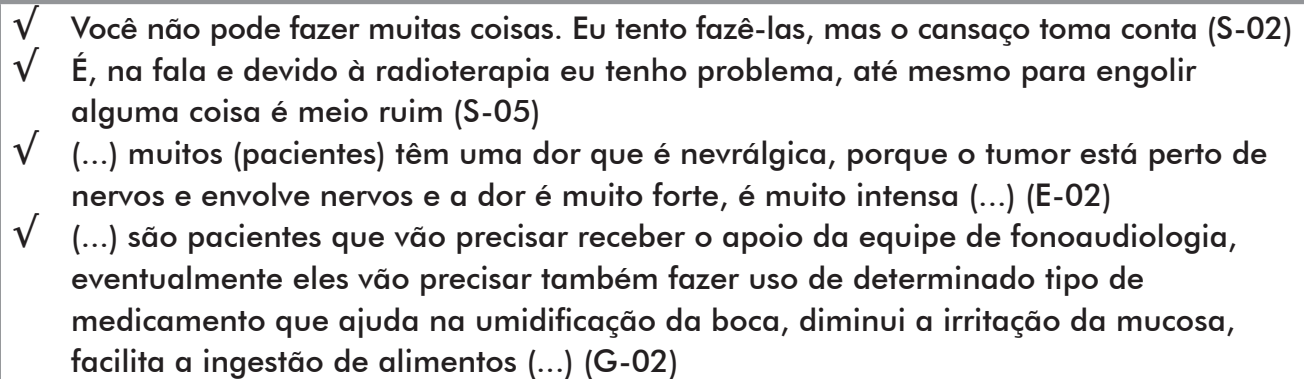 \\
\hline
\end{tabular}

Quadro 3. Depoimentos das subcategorias da categoria empírica "necessidades emocionais"

\begin{tabular}{|l|l|l|}
\hline \multicolumn{1}{|c|}{$\begin{array}{l}\text { Subcategorias } \\
\text { empíricas }\end{array}$} & \multicolumn{1}{c|}{\begin{tabular}{c} 
Trechos de depoimentos \\
\hline $\begin{array}{l}\text { Estratégias de } \\
\text { enfrentamento } \\
\text { ante a } \\
\text { possibilidade de } \\
\text { recidiva }\end{array}$
\end{tabular}} & $\sqrt{ } \begin{array}{l}\text { Não estou com medo, mas Deus não permita! Não quero de volta, mas se voltar, } \\
\text { pedirei ao médico que corte novamente (S-02) } \\
\text { Há quem termine o tratamento e, após 1 ano ou 2, volte e diga que a doença voltou } \\
\text { e eles choram junto conosco (E-02) }\end{array}$ \\
\hline $\begin{array}{l}\text { Suporte } \\
\text { psicológico ante } \\
\text { o diagnóstico }\end{array}$ & $\sqrt{ } \begin{array}{l}\text { Eu pensei que ia morrer em breve. Foi tudo o que pensei. Eu nem queria procurar } \\
\text { tratamento (S-05) } \\
\text { Eu achava que não era necessário, mas precisamos de ajuda. Às vezes, uma palavra } \\
\text { pode mudar nosso caminho. Uma palavra de uma pessoa bem-intencionada muda } \\
\text { nossa direção (S-04) } \\
\text { Oh, o medo do tratamento não, mas eu tive medo de morrer, porque eu acho que } \\
\text { todo mundo tem medo de morrer, né? (S-03) }\end{array}$ \\
\hline $\begin{array}{l}\text { Suporte } \\
\text { psicológico para } \\
\text { a sobrevivência }\end{array}$ & $\begin{array}{l}\sqrt{ } \\
\sqrt{ }\end{array}$ & $\begin{array}{l}\text { A angústia nunca te abandona, mas estou me sentindo mais forte (S-05) } \\
\text { (...) esses doentes só passam a receber um apoio, uma abordagem através da } \\
\text { psicóloga ou do psiquiatra, quando eles apresentam uma distorção comportamental } \\
\text { importante flagrada, identificada pelo médico assistente. Mas na minha opinião isso } \\
\text { deveria ser um caminho natural para todos esses pacientes, que são matriculados } \\
\text { aqui (G-02) } \\
\text { (...) teria que ter um tratamento psicológico. Um grupo de apoio (F-01) }\end{array}$ \\
\hline
\end{tabular}


Quadro 4. Depoimentos das subcategorias da categoria empírica "necessidades socioeconômicas"

\begin{tabular}{|c|c|}
\hline $\begin{array}{c}\text { Subcategorias } \\
\text { empíricas }\end{array}$ & Trechos dos depoimentos \\
\hline $\begin{array}{l}\text { Restabelecimento } \\
\text { da convivência } \\
\text { social }\end{array}$ & 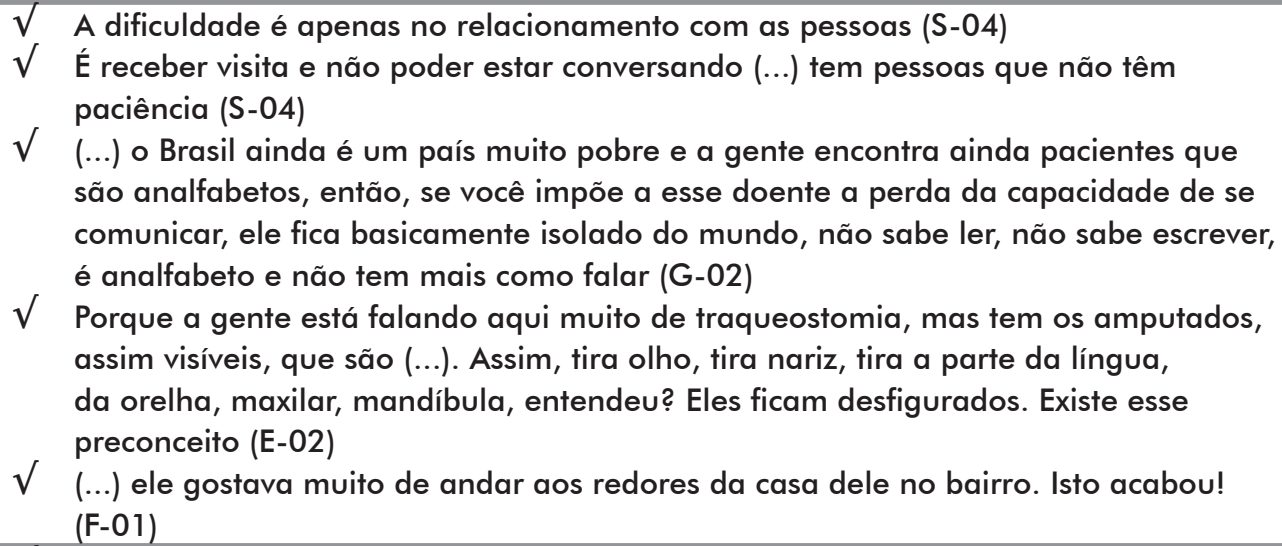 \\
\hline $\begin{array}{l}\text { Informações e } \\
\text { acessibilidade aos } \\
\text { direitos sociais }\end{array}$ & $\begin{array}{l}\sqrt{ } \text { Acho que erramos em termos do apoio social (G-01) } \\
\sqrt{ } \quad \begin{array}{l}\text { Faltou apenas mencionar o subsídio por doença [benefício concedido ao paciente } \\
\text { pelo governo brasileiro]; tudo o mais foi bom }(\mathrm{S}-05)\end{array}\end{array}$ \\
\hline
\end{tabular}

que mostram mudanças nos hábitos de vida, formas de alimentação e comunicação e relaçôes sociais.

As falas dos enfermeiros e dos médico-gestores, em uma abordagem técnico-descritiva, se igualam na exposição da complexidade e profundidade das alteraçôes físicas e da necessidade de apoio especializado e contínuo para as disfunçôes alimentares, reabilitação da fala, controle da dependência química de fumantes e controle da dor.

A melhoria dos tratamentos oncológicos e o consequente aumento no tempo de sobrevivência dos pacientes ${ }^{29}$ implicam em novos desafios para os serviços de saúde. Mesmo após o término do tratamento, ainda em unidades de atenção oncológica, os pacientes requerem açóes específicas que valorizem a sua qualidade de sobrevida e extrapolem os limites meramente clínicos do monitoramento da doença.

Entre as necessidades físicas abordadas pelos participantes, destacaram-se as necessidades de adaptaçóes do hábito alimentar e da capacidade de comunicação oral, bem como do controle da dependência química e manejo dos efeitos provocados pelo impacto da doença e seu tratamento.

As adaptaçôes do hábito alimentar estabelecem-se pelas repercussões da doença e seu tratamento no ato de comer e na experiência alimentar. $\mathrm{O}$ ato de comer é uma função complexa que envolve tanto uma necessidade biológica quanto uma satisfação gustativa, estética, emocional e social. Todas essas esferas são afetadas e impactadas. Os sentidos das falas indicam que os sobreviventes, por causa da disfagia, experimentam dificuldades para comer e beber ${ }^{15}$. Outras complicaçôes, como problemas dentários, trismo, perturbações sensoriais e alteraçóes no paladar, afetam a forma, o tipo e o ritual social da alimentação. Estas requerem novas formas de orientação nutricional, culinária e gastronômica para esses pacientes e seus familiares ${ }^{8,30}$.

As adaptaçóes da capacidade de comunicação oral remetem às repercussóes no processo de comunicação verbal. Os participantes apontam dificuldades na capacidade de expressão verbal em processos cotidianos de interação social. Essas limitações exigem novas soluções, pois a perspectiva ampliada de sobrevivência concebe um sobrevivente que, mesmo com doença ativa, mantém-se vivo no mundo, tentando retomar o cotidiano e planejar o futuro, apesar das incertezas quanto à cura. Uma alternativa de atenuação do quadro baseia-se na integração da paliação ${ }^{31,32}$, cuidados fisioterápicos e de reabilitação fonoaudiológica ${ }^{3,32-34}$.

Os participantes destacaram a importância do manejo dos efeitos provocados pelo impacto da doença $e$ seu tratamento. A terapia de câncer de cabeça e pescoço tem efeitos físicos debilitantes ${ }^{7}$ e o manejo desses pacientes é complexo, exigindo um cuidado contínuo de numerosa e especializada equipe multiprofissional. Embora as toxicidades agudas relacionadas à irradiação e aos agentes quimioterápicos possam diminuir logo após a cessação da terapia, o seu tempo de duração pode ser significativo. Além disso, podem ocorrer efeitos tardios e de longo prazo, que comprometem a qualidade de vida. Incluem-se nesse quadro doenças vasculares relacionadas a: radiação; xerostomia; fibrose dos músculos da deglutição; formação de cicatrizes; deterioração dentária; perda de sensaçôes gustativas e olfativas; perda auditiva; disfagia; dor; aspiração crônica; declínio nutricional e do bem- 
-estar geral, além de dificuldades funcionais que afetam a aparência ${ }^{3,4,34-36}$.

Outros aspectos do manejo e seguimento incluem o alto risco de recorrência da doença e incidência de doença metastática nos primeiros dois anos após o tratamento da doença primária. $\mathrm{O}$ foco central dos cuidados desse período é a identificação precoce de novos cânceres, gerenciamento dos efeitos tóxicos e tardios do tratamento e a provisão de cuidados primários de saúde como a prevenção e detecçáo precoce de doenças pulmonares e cardiovasculares $^{34}$.

Assim, no que se refere às necessidades físicas, cabe destacar que uma intervenção no âmbito global da sobrevivência que vise apenas a problemas clínicos é insuficiente. $\mathrm{O}$ cuidado a ser implementado deve atentar para as complexas relaçóes que se fazem entre as necessidades sociais e clínicas. A terapêutica deve ser singular e plural. Personalizada e humanizada em sua abordagem, conforme as necessidades específicas do sobrevivente; e plural em sua construção interdisciplinar, recorrendo às tecnologias e considerando a integralidade e a diversidade do sujeito ${ }^{37,38}$.

\section{NECESSIDADES EMOCIONAIS}

A categoria necessidades emocionais apresenta o sentido de necessidades como consequências do impacto psíquico da doença e seu tratamento. As necessidades de apoio emocional indicadas pelos entrevistados são relacionadas às questôes de suporte psicológico ante ao diagnóstico, suporte psicológico para a sobrevivência e estratégias de enfrentamento para possíveis recidivas.

Segundo Salz et al. ${ }^{36}, 50 \%$ dos sobreviventes de câncer são diagnosticados com alguma alteração psicológica. Panwar et al. $^{3}$ indicam que um terço dos pacientes apresenta distúrbios psicológicos significativos em longo prazo. Apesar disso, os distúrbios psiquiátricos graves são incomuns entre os sobreviventes de câncer.

Comparados com a população em geral, sobreviventes de cabeça e pescoço possuem um risco um pouco maior de apresentarem quadros de depressão ou transtorno de estresse pós-traumático, embora ainda faltem estudos que os caracterizem como efeitos de longo prazo ${ }^{36}$.

O suporte psicológico ante o diagnóstico pode amenizar o impacto da notícia. A experiência de angústia após um diagnóstico, e durante o tratamento de câncer, não é inesperada ou incomum. O estresse, nesses casos, é decorrente, basicamente, da ameaça existencial de uma doença potencialmente fatal e das incertezas que envolvem a tomada de decisão quanto aos rumos e resultados dos tratamentos realizados ${ }^{39}$. Considerando que o sofrimento emocional pode prejudicar a adesão aos tratamentos e piorar a evolução da doença ${ }^{40}$, o apoio psicológico se torna uma importante medida, em longo prazo, para o cuidado e a qualidade da sobrevivência dos pacientes de cabeça e pescoço.

O suporte psicológico para a sobrevivência é exigido pelos impactos tardios da doença. Segundo Stein et al. ${ }^{39}$, a identificação dos fatores que influenciam a trajetória e o equilíbrio psicológico do sobrevivente de câncer é um desafio. Para os autores, a resposta e o ajuste psicológico à experiência do câncer são influenciados pelo estresse, pelo fardo do adoecimento por câncer e pelos recursos disponíveis para o seu tratamento. Quanto maior o estresse e o fardo, maior o risco de efeitos psicológicos negativos a curto e longo prazos. Por outro lado, quanto maiores os recursos disponíveis, menor é esse risco. A compreensão do risco de um indivíduo em relação aos efeitos psicológicos negativos não deve se limitar apenas ao relato objetivo dos estressores colocados por sua experiência de câncer, mas explicar, também, a resposta subjetiva do indivíduo aos estressores presumidos. $\mathrm{O}$ acompanhamento psicológico, dessa forma, pode estimular a resiliência e ajudar o indivíduo a aprender a viver como sobrevivente ${ }^{40,41}$. Essas açōes também devem ser extensivas aos familiares, uma vez que comungam do mesmo sofrimento.

A estratégia de enfrentamento ante a possibilidade de recidiva se faz necessária, uma vez que um medo grave e persistente pode afetar significativamente a qualidade de vida, diminuir a capacidade funcional e aumentar o risco de abuso de tabaco ${ }^{3,39}$. Sentir que o futuro pode lhe ser ceifado é o elemento central que traduz o medo da recorrência do câncer. Muitos sobreviventes sem doença ativa experienciam medo e ansiedade quanto a essa possibilidade, demandando, para fins de controle, rastreio apropriado e terapia para auxiliar na redução dos sintomas ${ }^{42,43}$.

\section{NECESSIDADES SOCIOECONÔMICAS}

A categoria necessidades socioeconômicas apresenta o sentido de necessidades como consequências do impacto socioeconômico da doença e seu tratamento. As falas dos sobreviventes reivindicam a ampliação dos direitos sociais com açôes de suporte socioeconômico e reinserçáo laboral, com a finalidade de restabelecer e apoiar a sua existência social e individual. As falas dos enfermeiros e dos médico-gestores corroboram essas necessidades e reivindicações.

Os sentidos que emergem nessa categoria apontam para a complexidade das necessidades dos sobreviventes nos âmbitos social e econômico, exigindo açóes e políticas de: restabelecimento da convivência social; acesso às informações e aos direitos sociais.

$\mathrm{O}$ restabelecimento da convivência social envolve um duplo enfrentamento: as limitações impostas pela doença e o seu tratamento, combinadas com a identificação, 
mediação e promoção dos círculos de convivência dos sobreviventes. $\mathrm{O}$ enfrentamento do estresse, que a experiência do diagnóstico e tratamento do câncer traz, melhora quando o indivíduo consegue ser aceito e interage com o seu grupo social. $\mathrm{O}$ apoio social melhora, a curto e longo prazos, o ajustamento psicológico dos pacientes e sobreviventes de câncer ${ }^{44,45}$. No Brasil, esse tipo de apoio é previsto na Política Nacional para Prevenção e Controle do Câncer (PNPCC) ${ }^{46}$, que propóe a reabilitação do paciente oncológico como parte da assistência integral. Entretanto, tal dispositivo náo traz especificidades quanto às necessidades de reabilitaçấo social do paciente oncológico.

A necessidade de informaçóes e acessibilidade aos direitos sociais se impóe pelo excesso de burocracia, insuficiência de canais comunicativos e má qualidade e/ou falta das informaçóes sobre o tratamento clínico e pela requisição e obtenção de direitos previdenciários e sociais. Para pacientes com câncer e sobreviventes, o acesso a informaçôes precisas e compreensíveis sobre a doença, seu prognóstico, opções de tratamentos e os seus efeitos colaterais, disponibilidade e localização de serviços de apoio e o assessoramento quanto a direitos sociais e previdenciários são recursos valiosos ${ }^{15,47,48}$.

No Brasil, há iniciativas nesse campo. O Instituto Nacional de Câncer José Alencar Gomes da Silva (INCA) possui uma cartilha voltada para "Direitos sociais da pessoa com câncer” ${ }^{39}$, buscando promover uma fácil orientação e compreensão sobre o tema para os seus pacientes. No entanto, são necessárias outras iniciativas e outros materiais didáticos que integrem informaçôes econômicas e sociais e considerem as necessidades e o nível sociocultural do público-alvo. Cabe destacar, entretanto, que o conjunto de benefícios e direitos sociais garantidos nas políticas públicas contemplam apenas os pacientes oncológicos com doença ativa em suas etapas de diagnóstico e tratamento. Eles ainda não abrangem as peculiaridades dos sobreviventes e de seus familiares que seguem com vulneraçóes econômicas e sociais.

\section{CONCLUSÃO}

A sobrevivência ao câncer é cada vez mais reconhecida como uma entidade distinta dentro do continuum do cuidado e do controle do câncer. Como ponto de transiçáo do tratamento imediato para um acompanhamento de longo prazo, essa fase é um período importante para todo um planejamento de toda uma série de açôes preventivas relacionadas ao rastreamento de novos tipos de câncer, tais como gerenciamento de comorbidades tardias e de longo prazo, reabilitação, promoção da saúde, emancipação e empoderamento social.
Os sobreviventes de câncer de cabeça e pescoço brasileiros enfrentam, no pós-tratamento, vários desafios. Essa situação se consubstancia em uma ampla gama de necessidades físicas, emocionais e socioeconômicas que, não só os acompanham, como afetam aos seus familiares e cuidadores. As necessidades mais prementes a serem atendidas são: acompanhamento psicológico para pacientes e familiares; e maior necessidade de informaçóes individualizadas sobre benefícios sociais e previdenciários.

Há necessidade de mais estudos nacionais de forma a aumentar o conhecimento das especificidades da nossa populaçáo. Apesar disso, mesmo com deficiência de informaçôes, verificou-se que há uma necessidade urgente de formação e aperfeiçoamento de políticas públicas focadas para a fase do pós-tratamento do câncer que contemplem, para além das questóes físicas, as prementes dimensóes das necessidades emocionais e socioeconômicas.

A principal limitação deste estudo, de natureza exploratória e descritiva, está em ter sido realizado em um cenário limitado. Apesar de ser possível a realização de estudos qualitativos com pequenas amostras, estes não podem ser generalizados. Os resultados obtidos, embora significativos pelo poder da informação contida nos depoimentos, indicam a necessidade de realização de estudos adicionais que incluam, preferencialmente, mais indivíduos com um tempo de diagnóstico e tratamento entre cinco e dez anos antes das entrevistas. Ação que permitirá ampliar a compreensão e o dimensionamento sobre as necessidades de cuidados desse grupo.

\section{CONTRIBUIÇÕES}

Carolina de Menezes Rabello contribuiu substancialmente na concepção ou no planejamento do estudo; na obtenção, análise e/ou interpretação dos dados; e na redaçâo. Rildo Pereira da Silva e Antonio Tadeu Cheriff dos Santos contribuíram substancialmente no planejamento do estudo; na análise e/ou interpretação dos dados; e na revisão crítica. Fernando Lopes Tavares de Lima e Liz Maria de Almeida contribuíram substancialmente na revisão crítica. Todos os autores aprovaram a versão final a ser publicada.

\section{DECLARAÇÃO DE CONFLITO DE INTERESSES}

Nada a declarar.

\section{FONTES DE FINANCIAMENTO}

Não há. 


\section{REFERÊNCIAS}

1. Goon PK, Stanley MA, Ebmeyer J, et al. HPV \& head and neck cancer: a descriptive update. Head Neck Oncol. 2009;1:36. doi: https://doi.org/10.1186/17583284-1-36

2. Instituto Nacional de Câncer José de Alencar Gomes da Silva. Estimativa 2020: incidência de câncer no Brasil. Rio de Janeiro: INCA; 2019.

3. Panwar A, Cheung VWF, Lydiatt WM. Supportive care and survivorship strategies in management of squamous cell carcinoma of the head and neck. Hematol Oncol Clin North Am. 2015;29(6):1159-68. doi: https://doi. org/10.1016/j.hoc.2015.07.010

4. Curioni OA, Chagas JFS, Marcucci M, et al. Valor do protocolo diagnóstico e terapêutico no tratamento do câncer da cabeça e pescoço no Departamento de Cirurgia de Cabeça e Pescoço e Otorrinolaringologia do Hospital Heliópolis, São Paulo. Rev Bras Cir Cabeça Pescoço [Internet]. 2012 [acesso 2021 jul 27];41(4):159-62. Disponível em: http:/www.sbccp.org.br/wp-content/ uploads/2014/11/REVISTA-SBCCP-41-4-artigo-01.pdf

5. Mullan F. Seasons of survival: reflections of a physician with cancer. N Engl J Med. 1985;313(4):270-3. doi: https://doi.org/10.1056/NEJM198507253130421

6. Taylor JC, Terrell JE, Ronis DL, et al. Disability in patients with head and neck cancer. Arch Otolaryngol Head Neck Surg. 2004;130(6):764-9. doi: https://doi. org/10.1001/archotol.130.6.764

7. Abbott DM, Cohen EEW, Sadeghi N, et al. Considering the survivorship care needs of head and neck cancer survivors. Oral Oncol. 2016;57:61-2. doi: https://doi. org/10.1016/j.oraloncology.2016.04.012

8. Simcock R, Simo R. Follow-up and survivorship in head and neck cancer. Clin Oncol (R Coll Radiol). 2016;28(7):451-8. doi: https://doi.org/10.1016/j. clon.2016.03.004

9. Hewitt M, Greenfield S, Stovall, editors. From cancer patient to cancer survivor: lost in transition. Washington, DC: National Academies Press; c2006.

10. Halpern MT, Argenbright KE. Evaluation of effectiveness of survivorship programmes: how to measure success? Lancet Oncol. 2017;18(1):e51-e59. doi: https://doi. org/10.1016/S1470-2045(16)30563-0

11. Cohen EE, LaMonte SJ, Erb NL, et al. American Cancer Society head and neck cancer survivorship care guideline. CA Cancer J Clin. 2016;66(3):203-39. doi: https://doi. org/10.3322/caac. 21343

12. Nekhlyudov L, Lacchetti C, Davis NB, et al. Head and neck cancer survivorship care guideline: American Society of Clinical Oncology Clinical Practice guideline endorsement of the American Cancer Society Guideline. J Clin Oncol. 2017;35(14):1606-21. doi: https://doi. org/10.1200/JCO.2016.71.8478
13. Birken SA, Mayer DK, Weiner BJ. Survivorship care plans: prevalence and barriers to use. J Cancer Educ. 2013;28(2):290-6. doi: https://doi.org/10.1007/s13187013-0469-x

14. Viswanathan M, Halpern M, Swinson Evans T, et al. Models of cancer survivorship care. Rockville (MD): Agency for Healthcare Research and Quality (US); 2014 Mar. (Technical Briefs; no 14).

15. Manne S, Hudson SV, Baredes S, et al. Survivorship care experiences, information, and support needs of patients with oral and oropharyngeal cancer. Head Neck. 2016;38(Suppl 1):E1935-46. doi: https://doi. org/10.1002/hed.24351

16. Worrell E, Worrell L, Bisase B. Care of long-term survivors of head and neck cancer after treatment with oral or facial prostheses, or both. Br J Oral Maxillofac Surg. 2017;55(7):685-90. doi: https://doi.org/10.1016/j. bjoms.2017.04.014

17. Pinto GP, Mont'alverne DGB. Neoplasias de cabeça e pescoço: impactos funcionais e na qualidade de vida. Rev Bras Cir Cabeça Pescoço [Internet]. 2015 [acesso 2020 jul 22];44(3):152-6. Disponível em: http://www.sbccp. org.br/wp-content/uploads/2015/07/Rev-SBCCP-443-artogo-09.pdf

18. Minayo MCS. O desafio do conhecimento: pesquisa qualitativa em saúde. 11. ed. São Paulo: Hucitec; 2008.

19. Gomes R. Pesquisa qualitativa em saúde. São Paulo: Instituto Sírio-Libanês de Ensino e Pesquisa; 2014.

20. Laverty SM. Hermeneutic phenomenology and phenomenology: a comparison of historical and methodological considerations. Int J Qual Methods. 2003;2(3):21-35. doi: https://doi. org/10.1177/160940690300200303

21. Breilh J. Epidemiologia economia, política e saúde. [São Paulo]: UNESP; 1991. (Saúde em debate; 45).

22. Minayo MCS, organizadora. Pesquisa social: teoria, método e criatividade. Petrópolis: Vozes; 2009. Capítulo 4, Gomes R. Análise e interpretação de dados de pesquisa qualitativa; p. 79.

23. Braun V, Clarke V. What can "thematic analysis" offer health and wellbeing researchers? Int J Qual Stud Health Well-being. 2014;9:26152. doi: https://doi.org/10.3402/ qhw.v9.26152

24. Gadamer HG. Truth and method. 2nd ed. New York: Continuum; 1998. (Bloomsbury revelations).

25. Ricouer P. Interpretação e ideologias. Rio de Janeiro: Francisco Alves; 1990.

26. Tan H, Wilson A, Olver I. Ricoeur's theory of interpretation: an instrument for data interpretation in hermeneutic phenomenology. Int J Qual Methods. 2009;8(4):1-15. doi: https://doi.org/10.1177/160940690900800401

27. Habermas J. Theory and practice. Boston: Beacon Press; 1973. 
28. Habermas J. Dialética e hermenêutica: para a crítica da hermenêutica de Gadamer. Porto Alegre: L\&PM; 1987.

29. Oliveira RAA, Araujo JS, Conceiçáo VM, et al. Sobrevivência ao câncer: o desembrulhar dessa realidade. Ciênc Cuid Saúde. 2015;14(4):1602-8. doi: https://doi. org/10.4025/cienccuidsaude.v14i4.27445

30. Ganzer H, Touger-Decker R, Byham-Gray L, et al. The eating experience after treatment for head and neck cancer: a review of the literature. Oral Oncol. 2015;51(7):634-42. doi: https://doi.org/10.1016/j. oraloncology.2015.04.014

31. Carvalho RT, Parsons HA, organizadores. Manual de cuidados paliativos ANCP. 2. ed. amp. rev. Rio de Janeiro: Academia Nacional de Cuidados Paliativos; 2012.

32. Nguyen NA, Ringash J. Head and neck cancer survivorship care: a review of the current guidelines and remaining unmet needs. Curr Treat Options Oncol. 2018;19(8):44. doi: https://doi.org/10.1007/s11864018-0554-9

33. Campos RJDS, Leite ICG. Qualidade de vida e voz pós-radioterapia: repercussóes para a fonoaudiologia. Rev CEFAC. 2010;12(4):671-7. doi: https://doi. org/10.1590/S1516-18462010005000038

34. Shapiro CL. Cancer survivorship. N Engl J Med. 2018;379(25):2438-50. doi: https://doi.org/10.1056/ NEJMra1712502

35. Reid K, Soundy A. A qualitative study examining the illness narrative master plots of people with head and neck cancer. Behav Sci (Basel). 2019;9(10):110. doi: https://doi.org/10.3390/bs9100110

36. Salz T, McCabe MS, Oeffinger KC, et al. A head and neck cancer intervention for use in survivorship clinics: a protocol for a feasibility study. Pilot Feasibility Stud. 2016;2:23. doi: https://doi.org/10.1186/s40814-0160061-3

37. Pinto DM, Jorge MSB, Pinto AGA, et al. Projeto terapêutico singular na produção do cuidado integral: uma construção coletiva. Texto Contexto Enferm. 2011;20(3):493-302. doi: https://doi.org/10.1590/ S0104-07072011000300010

38. Ministério da Saúde (BR). HumanizaSUS: Política Nacional de Humanização: a humanização como eixo norteador das práticas de atenção e gestâo em todas as instâncias do SUS. Brasília, DF: Ministério da Saúde; 2004.

39. Stein KD, Syrjala KL, Andrykowski MA. Physical and psychological long-term and late effects of cancer. Cancer. 2008;112(11 Suppl):2577-92. doi: https://doi. org/10.1002/cncr.23448

40. Veit MT, Carvalho VA. Psico-oncologia: um novo olhar para o câncer. Mundo Saúde. 2010;34(4):526-30. doi: https://doi.org/10.15343/0104-7809.20104526530

41. Saavedra Guajardo E, Villalta Paucar M. Medición de las características resilientes: un estudio comparativo en personas entre 15 y 65 años. Liber. 2008;14(14):32-40. Spanish.

42. Humphris GM, Rogers S, McNally D, et al. Fear of recurrence and possible cases of anxiety and depression in orofacial cancer patients. Int J Oral Maxillofac Surg. 2003;32(5):486-91. doi: https://doi.org/10.1016/ S0901-5027(03)90399-1

43. Ghazali N, Cadwallader E, Lowe D, et al. Fear of recurrence among head and neck cancer survivors: longitudinal trends. Psychooncology. 2013;22(4):80713. doi: https://doi.org/10.1002/pon.3069

44. Giuliani M, Milne R, McQuestion M, et al. Partner's survivorship care needs: an analysis in head and neck cancer patients. Oral Oncol. 2017;71:113-21. doi: https://doi.org/10.1016/j.oraloncology.2017.06.011

45. Semple C, Parahoo K, Norman A, et al. Psychosocial interventions for patients with head and neck cancer. Cochrane Database Syst Rev. 2013;(7):CD009441. doi: https://doi.org/10.1002/14651858.CD009441.pub2

46. Ministério da Saúde (BR). Portaria de Consolidação $n^{\circ} 2$, de 28 de setembro de 2017. Consolidação das normas sobre as políticas nacionais de saúde do Sistema Único de Saúde [Internet]. Diário Oficial da Uniāo. Brasília, DF. 2017 out 3 [acesso 2020 jul 10];Seção Suppl:61. Disponível em: http://bvsms.saude.gov.br/ bvs/saudelegis/gm/2017/prc0002_03_10_2017.html

47. Ghazali N, Roe B, Lowe D, et al. Patients concerns inventory highlights perceived needs and concerns in head and neck cancer survivors and its impact on health-related quality of life. Br J Oral Maxillofac Surg. 2015;53(4):371-9. doi: https://doi.org/10.1016/j. bjoms.2015.01.022

48. van Linden van den Heuvell C, van Zuuren F, Wells $\mathrm{M}$, et al. Paradigm shift in head and neck oncology patient management. J Otolaryngol Head Neck Surg. 2017;46:57. doi: https://doi.org/10.1186/s40463-0170229-8

49. Instituto Nacional de Câncer José Alencar Gome da Silva. Direitos sociais da pessoa com câncer: orientaçóes aos usuários. 5. ed. Rio de Janeiro: INCA; 2020.

Recebido em 28/9/2020 Aprovado em 19/2/2021 